\title{
Circulating Tumour DNA to Guide Treatment of Gastrointestinal Malignancies
}

\author{
Yat Hang To ${ }^{a, b}$ Belinda Lee ${ }^{a-c, e}$ Hui-Li Wong ${ }^{a, b}$ Peter Gibbs ${ }^{a, d, e}$ \\ Jeanne Tie ${ }^{a, b}, d, e$ \\ a Division of Systems Biology and Personalised Medicine, Walter and Eliza Hall Institute of Medical Research, \\ Parkville, VIC, Australia; ${ }^{b}$ Department of Medical Oncology, Peter MacCallum Cancer Centre, Melbourne, VIC, \\ Australia; ${ }^{\mathrm{C}}$ Department of Medical Oncology, The Northern Hospital, Epping, VIC, Australia; ${ }^{\mathrm{d}}$ Department of \\ Medical Oncology, Western Health, Footscray, VIC, Australia; ${ }^{~}$ Faculty of Medicine, Dentistry and Health Sciences, \\ University of Melbourne, Melbourne, VIC, Australia
}

\section{Keywords}

Liquid biopsy - Circulating tumour DNA · ctDNA ·

Gastrointestinal cancer · Colorectal cancer

\begin{abstract}
Background: Gastrointestinal cancers are among the most common cancers worldwide and account for a high proportion of cancer-related mortality. Advancements to improve outcomes are constrained by the lack of biomarkers that can offer early diagnostic and prognostic information as traditional serological tumour markers and conventional imaging approaches are not able to provide early information regarding disease recurrence and treatment outcomes. Recent advances in technology have allowed the detection of circulating tumour DNA (ctDNA) in plasma, nucleic acid fragments released into the circulation from primary or metastatic lesions undergoing apoptosis and necrosis. A growing body of evidence has emerged supporting the use of ctDNA in many aspects of cancer care. Summary: This review focuses on the potential role of ctDNA in the management of patients with gastrointestinal cancers including colorectal, pancreatic, and upper gastrointestinal cancers. In this review, we discuss its possible utility in screening, detection of minimal residual disease and prognostication, longitudinal surveillance, and identification of therapeutic targets and resistance incorporating recent literature and
\end{abstract}

ongoing randomised clinical trials. Key Messages: ctDNA has substantial potential as a clinically useful marker in the management of gastrointestinal cancers from cancer screening through to treatment of advanced disease.

(c) 2020 S. Karger AG, Base

\section{Introduction}

Cell-free deoxyribonucleic acid (cfDNA) are DNA fragments that can be found circulating in plasma and other bodily fluids. In 1989, Stroun et al. [1] were the first to demonstrate that a proportion of cfDNA detected in cancer patients was derived from cancer cells. Now widely known as circulating tumour DNA (ctDNA), these tumour-derived genetic fragments are thought to be released from primary and metastatic sites of cancer, by apoptosis or necrosis, as well as from circulating tumour cells [2]. Since its discovery, an evolving field dedicated to the detection and interrogation of ctDNA as a biomarker in early diagnosis, detection of recurrence, and its potential role in driving treatment decision-making has emerged.

Broadly, there are two different approaches to ctDNA detection [3]. The first is a tumour-informed approach in a patient with known malignancy, requiring prior knowledge of target mutations specific to the tumour, usually 
Table 1. Proposed applications of circulating tumour DNA (ctDNA) in clinical practice

\begin{tabular}{ll}
\hline Application & Proposed utility \\
\hline Screening & $\begin{array}{l}\text { - Identifying biomarkers that could be used as a cancer screening tool to detect early-stage and } \\
\text { asymptomatic cancers allowing earlier intervention }\end{array}$ \\
\hline $\begin{array}{l}\text { Detection of minimal } \\
\text { residual disease }\end{array}$ & $\begin{array}{l}\text { - Detection of ctDNA after curative intent surgery has been shown to correlate with an increased } \\
\text { risk of relapse, thus offering prognostic information } \\
\text { - Allowing a risk-adjust approach to adjuvant treatment, including escalating or de-escalating } \\
\text { treatment intensity based on ctDNA analysis }\end{array}$ \\
\hline $\begin{array}{l}\text { Longitudinal } \\
\text { surveillance }\end{array}$ & $\begin{array}{l}\text { - Serial monitoring of ctDNA during and after adjuvant treatment to allow early identification of } \\
\text { relapse or progression prior to clinical or radiological manifestation }\end{array}$ \\
\hline $\begin{array}{l}\text { Identifying therapeutic } \\
\text { targets and resistance }\end{array}$ & $\begin{array}{l}\text { - Identifying presence of clinically actionable mutations; especially beneficial in cases where tumour } \\
\text { tissue cannot be easily retrieved and may better reflect tumour heterogeneity } \\
\text { - Identifying mechanisms of resistance to targeted treatment } \\
\text { - As an early measure of tumour response to treatment }\end{array}$ \\
\hline
\end{tabular}

assessed through whole exome sequencing or targeted next-generation sequencing of tumour tissue, to inform what mutation(s) are to be detected in the plasma. Methods that adopt this tumour-informed approach include targeted sequencing platforms modified with molecular barcoding methods and error-suppression algorithms such as Safe-Seqs (Safe Sequencing System) and CAPPSeq (CANcer Personalized Profiling by deep Sequencing) as well as PCR platforms such as droplet digital PCR and BEAMing. The second approach is tumour agnostic (or non-tumour informed), analysing plasma samples for mutations without prior analysis of tumour tissue, targeting frequently mutated cancer genes (e.g., KRAS, EGFR), epigenetic changes, and other analytes in plasma. Although both approaches have been adopted in clinical trials, to date there is limited data to directly compare the two approaches and the optimal assay remains to be defined.

As an easily accessible biomarker, the potential role of ctDNA in clinical cancer diagnosis and treatment has been widely speculated and continues to be explored. Proposed applications are summarised in Table 1.

In this review article, we aim to explore the current literature surrounding the clinical utility of ctDNA in gastrointestinal malignancies.

\section{Colorectal Cancer}

\section{Screening}

The Australian National Bowel Cancer Screening Program provides biennial immunochemical faecal occult blood test screening for people aged 50-74 years. While proven to reduce cancer incidence and cancer deaths, only $42 \%$ of invited participants complete the test [4]. Notably, it has been demonstrated that patients prefer a blood test as the chosen modality of non-invasive screening for colorectal cancers (CRC) suggesting that incorporation of ctDNA testing may improve compliance [5]. Our group used a tumour-informed approach to analyse patients with newly diagnosed CRC, detecting plasma ctDNA in $46 \%$ of patients with stage I cancers, demonstrating that ctDNA is present even in early-stage cancers [6]. Rates of detection were higher in locally advanced rectal cancers (80\%) [6]. Similarly, Bettegowda et al. [7] analysed 640 patients with various cancer types; ctDNA was detected in $73 \%$ of patients with a localised CRC.

The aforementioned studies used a tumour-informed approach, a strategy which is not feasible when screening a well population. In this context, Cohen et al. [8] developed the multi-analyte CancerSEEK test, a blood-based test combining tumour-agnostic ctDNA detection and circulating protein biomarker assessment. Median sensitivity amongst multiple eight cancer types including liver, stomach, pancreas, oesophagus, and colorectum was 70\% $\left(p<10^{-96}\right)$. This was lower for stage I cancers $(43 \%)$ but varied between cancer types (liver cancer $100 \%$, oesophageal $20 \%$ ). The primary site of the cancer was able to be localised to one of two anatomic sites in a median of $83 \%$ of these patients $\left(p<10^{-77}\right)$. Further studies adopting such a tumour-agnostic approach to ctDNA screening in large asymptomatic populations are needed to validate this approach. Impacts on health economics, potential harm from unnecessary interventions, and patients' psychological health also need to be addressed before ctDNA can be routinely incorporated into CRC screening.

\section{Detection of Minimal Residual Disease}

ctDNA has been demonstrated to detect minimal residual disease, defined as the occult presence of cancer cells after curative intent treatment that will eventually lead to relapse. In a prospective cohort of 230 patients 
Table 2. Examples of ongoing ctDNA-guided randomised clinical trials in localised gastrointestinal malignancies

\begin{tabular}{|c|c|c|c|c|c|}
\hline Trial name & Country & Study population & $\begin{array}{l}\text { Target } \\
\text { sample size }\end{array}$ & Study design & $\begin{array}{l}\text { Primary } \\
\text { endpoint }\end{array}$ \\
\hline $\begin{array}{l}\text { Colorectal cancer } \\
\text { DYNAMIC II } \\
\text { (ACTRN12615000381583) }\end{array}$ & Australia & $\begin{array}{l}\text { Curatively resected stage II } \\
\text { colon cancer }\end{array}$ & 450 & $\begin{array}{l}\text { Randomised controlled; randomised 2:1 to ctDNA-informed or standard } \\
\text { of care adjuvant chemotherapy } \\
\text { Intervention: } \\
\text { - ctDNA arm: adjuvant chemotherapy if positive, surveillance if negative } \\
\text { - Standard of care arm: at investigator's discretion }\end{array}$ & $\begin{array}{l}\text { 3-year } \\
\text { recurrence- } \\
\text { free survival }\end{array}$ \\
\hline $\begin{array}{l}\text { CIRCULATE } \\
(\text { NCT04089631) }\end{array}$ & $\begin{array}{l}\text { Germany, } \\
\text { Austria, } \\
\text { Sweden }\end{array}$ & $\begin{array}{l}\text { Curatively resected stage } \\
\text { colon and high rectum } \\
\text { adenocarcinoma }\end{array}$ & 4,812 & $\begin{array}{l}\text { Phase III randomised controlled; ctDNA-positive patients randomised } \\
\text { 2:1 to adjuvant chemotherapy (capecitabine } \pm \text { oxaliplatin) or } \\
\text { trial-scheduled follow-up; ctDNA-negative patients randomised 1:4 } \\
\text { to trial-scheduled follow-up or standard of care follow-up }\end{array}$ & $\begin{array}{l}\text { Disease-free } \\
\text { survival }\end{array}$ \\
\hline $\begin{array}{l}\text { PRODIGE } 70- \\
\text { CIRCULATE } \\
(\text { NCT04120701) }\end{array}$ & France & $\begin{array}{l}\text { Curatively resected stage II } \\
\text { colon and high rectum } \\
\text { adenocarcinoma }\end{array}$ & 1,980 & $\begin{array}{l}\text { Phase III randomised controlled; randomised to chemotherapy } \\
\text { arm (FOLFOX6m; all patients in this arm will have ctDNA tested), } \\
\text { trial-scheduled follow-up or standard of care follow-up }\end{array}$ & $\begin{array}{l}\text { 3-year disease- } \\
\text { free survival }\end{array}$ \\
\hline $\begin{array}{l}\text { NRG-GI005 (COBRA) } \\
\text { (NCT04068103) }\end{array}$ & United States & $\begin{array}{l}\text { Curatively resected stage } \\
\text { IIA adenocarcinoma of the } \\
\text { colon and appropriate for } \\
\text { active surveillance (i.e., no } \\
\text { adjuvant chemotherapy) }\end{array}$ & 1,408 & $\begin{array}{l}\text { Phase II/III randomised; patients in experiment arm II assigned to } \\
\text { ctDNA positive (Group 1) and ctDNA negative (Group 2) } \\
\text { Intervention: } \\
\text { - Group 1: FOLFOX or XELOX at investigator's discretion. } \\
\text { - Group 2: active surveillance }\end{array}$ & $\begin{array}{l}\text { Rate of ctDNA } \\
\text { "clearance"; } \\
\text { 3-year } \\
\text { recurrence- } \\
\text { free survival }\end{array}$ \\
\hline $\begin{array}{l}\text { DYNAMIC III } \\
\text { (ACTRN12617001566325) }\end{array}$ & Australia & $\begin{array}{l}\text { Curatively resected stage III } \\
\text { colon cancer }\end{array}$ & 1,000 & $\begin{array}{l}\text { Phase II/III randomised; randomised 1:1 to ctDNA-informed or standard } \\
\text { of care adjuvant chemotherapy } \\
\text { Intervention: } \\
\text { - ctDNA arm: escalation of planned adjuvant chemotherapy regimen if } \\
\text { ctDNA positive; de-escalation if negative } \\
\text { - Standard of care arm: at investigator's discretion }\end{array}$ & $\begin{array}{l}\text { 3-year } \\
\text { recurrence- } \\
\text { free survival }\end{array}$ \\
\hline $\begin{array}{l}\text { COLUMBIA } 2 \\
\text { (NCT04145193) }\end{array}$ & International & $\begin{array}{l}\text { Resected stage II and III, } \\
\text { microsatellite-stable, } \\
\text { ctDNA-positive colorectal } \\
\text { cancer }\end{array}$ & 160 & $\begin{array}{l}\text { Phase II study; randomising post-surgery ctDNA-positive patient 1:1:1:1 } \\
\text { to standard of care adjuvant chemotherapy or chemotherapy and novel } \\
\text { oncology therapies } \\
\text { Intervention: } \\
\text { - mFOLFOX6 } \\
\text { - mFOLFOX + durvalumab } \\
\text { - mFOLFOX, durvalumab + oleclumab } \\
\text { - mFOLFOX6, durvalumab + monalizumab }\end{array}$ & $\begin{array}{l}\text { Rate of ctDNA } \\
\text { "clearance" }\end{array}$ \\
\hline $\begin{array}{l}\text { DYNAMIC Rectal } \\
\text { (ACTRN12617001560381) }\end{array}$ & Australia & $\begin{array}{l}\text { Locally rectal cancer treated } \\
\text { with pre-operative long- } \\
\text { course chemoradiation and } \\
\text { surgery }\end{array}$ & 408 & $\begin{array}{l}\text { Randomised controlled; randomised 2:1 to ctDNA-informed or standard } \\
\text { of care adjuvant chemotherapy } \\
\text { Intervention: } \\
\text { - ctDNA arm: } 4 \text { months of adjuvant fluoropyrimidine-based } \\
\text { chemotherapy if ctDNA positive or negative with high-risk features } \\
\text { (based on standard pathology risk assessment) } \\
\text { - Standard of care arm: at investigator's discretion }\end{array}$ & $\begin{array}{l}\text { 3-year } \\
\text { recurrence- } \\
\text { free survival }\end{array}$ \\
\hline $\begin{array}{l}\text { Pancreas cancer } \\
\text { DYNAMIC Pancreas } \\
\text { (ACTRN12618000335291) }\end{array}$ & Australia & $\begin{array}{l}\text { Locally advanced } \\
\text { adenocarcinoma of the } \\
\text { pancreas treated with pre- } \\
\text { operative chemotherapy } \\
\text { and surgery }\end{array}$ & 438 & $\begin{array}{l}\text { Phase II/III randomised controlled; if received neoadjuvant } \\
\text { FOLFIRINOX chemotherapy, randomised 1:1 to standard of care } \\
\text { (Cohort A) or ctDNA-informed (Cohort B) adjuvant chemotherapy. } \\
\text { If curative surgery alone, enrolled to Cohort C (observational arm, } \\
\text { non-randomised) } \\
\text { Intervention: } \\
\text { - Cohort A: at investigator's discretion } \\
\text { - Cohort B: } 4-6 \text { months of gemcitabine-based doublet chemotherapy if } \\
\text { ctDNA positive; } 3 \text { months of FOLIFIRINOX if negative } \\
\text { - Cohort C: escalated, de-escalated, or switched from intended adjuvant } \\
\text { chemotherapy based on ctDNA result }\end{array}$ & $\begin{array}{l}2 \text {-year } \\
\text { recurrence- } \\
\text { free survival }\end{array}$ \\
\hline $\begin{array}{l}\text { Solid organ cancers } \\
\text { NCT03832569 }\end{array}$ & United States & $\begin{array}{l}\text { MSI-H solid organ cancers } \\
\text { with positive ctDNA within } \\
4-5 \text { months after } \\
\text { completion of standard of } \\
\text { care therapy (surgery, } \\
\text { chemotherapy, radiation) }\end{array}$ & 48 & $\begin{array}{l}\text { Phase } 2 \text { randomised, double-blind study; randomised to either } \\
\text { pembrolizumab or placebo }\end{array}$ & $\begin{array}{l}\text { Disease-free } \\
\text { survival }\end{array}$ \\
\hline
\end{tabular}

with resected stage II colon cancers, we showed that patients with detectable ctDNA post-surgery have markedly reduced recurrence-free survival compared to those that did not (HR 18; 95\% CI 7.9-40) [9]. The prognostic impact of post-surgery ctDNA was similarly observed in patients with locally advanced rectal cancer treated with neoadjuvant chemoradiotherapy and surgery (3-year
RFS 33 vs. $87 \%$, positive versus negative ctDNA) [10]. Reinert et al. [11] reported similar data in patients with stage II to III CRC, demonstrating that ctDNA detection was associated with reduced RFS (HR 7.2; 95\% CI 2.719.0). In all three studies, multivariable analysis confirmed that ctDNA was an independent biomarker of relapse. 
Based on these studies, it is clear that ctDNA can be used to identify patients at high risk of relapse post-curative intent treatment. For stage II colon cancers, where the survival impact of chemotherapy remains uncertain, ctDNA detection post-surgery could define a cohort likely to benefit from adjuvant chemotherapy (ACT). Various international studies (Table 2) are currently exploring either a ctDNA-based adjuvant strategy, randomising patients to a ctDNA informed arm where treatment is based on ctDNA result versus standard-of-care adjuvant treatment (e.g., DYNAMIC and COBRA), or a ctDNAby-treatment interaction design, in which ctDNA-positive patients are randomised to investigational therapy versus control and ctDNA-negative patients can undergo the same randomisation or are offered standard treatment (e.g., CIRCULATE). For stage III cancers, ctDNA could be used to stratify risk, patients with detectable ctDNA being candidates for more intense adjuvant therapy and surveillance, whereas the low-risk patients with undetectable ctDNA may be managed with de-escalated treatment without compromising survival outcomes. The currently recruiting DYNAMIC-III trial is exploring this approach. The ability of post-operative ctDNA positivity to enrich for very-high-risk patients could also help expedite novel drug development in the adjuvant setting. Several industry-led trials have begun using this strategy.

\section{Longitudinal Surveillance}

Evaluation of ctDNA dynamics during and following ACT may serve as an early indicator of treatment outcome. It has been shown across both stage II and III CRCs that ctDNA positivity after ACT is associated with poorer RFS [11, 12]. This appears to demonstrate that persistent detection of ctDNA post-ACT reflects presence of micrometastatic disease, which ultimately is the source of clinical recurrence. Serial monitoring during post-treatment surveillance also appears to predict relapse with significant lead time over serological CEA monitoring, ranging from 100 days to 8.7 months across different studies. These studies tested ctDNA at different time points and did not perform imaging with each ctDNA analysis, likely accounting for the variation in lead time. Despite this, these studies illustrate that earlier detection of recurrence may be possible with incorporation of longitudinal ctDNA testing into surveillance programs. But whether bringing forward the diagnosis of recurrence impacts survival outcomes is a key unanswered question.

In the metastatic setting, ctDNA dynamics may reflect treatment response. In a study of 53 patients with metastatic CRC (mCRC) receiving first-line chemotherapy, a 10 -fold reduction in ctDNA mutant allele fraction 2 weeks after commencing chemotherapy correlated with a radiological response at $8-10$ weeks [13]. A later study demonstrated that changes in ctDNA concentration even as early as 1 week after initiation of chemotherapy also predicted for response and PFS [14]. Therefore, ctDNA monitoring may allow early prediction of response or progression, but how this should guide treatment decision-making remains uncertain.

\section{Identifying Therapeutic Targets and Resistance}

The standard treatment of mCRC that are wild-type for KRAS and NRAS includes EGFR blockade, although benefit is limited in patients with a right-sided primary tumour. Current assessment of mutation status is based on analysis of tumour tissue, but ctDNA could provide an easier method of detection. Many studies have shown a high concordance ( $~ 90 \%)$ between RAS status in matched cfDNA and tumour tissue samples, with sensitivities and specificities ranging from 70 to $96 \%$ and 83 to $98 \%$, respectively [15]. Reasons for the failure to detect RAS mutation in the plasma have been shown to be largely due to the lack of ctDNA being released by the tumour, which is associated with low tumour burden, peritoneal, nodal, or lung metastases, and mucinous histology [15]. Several retrospective studies have also observed similar outcomes in patients with mCRC who were treated with antiEGFR-based regimens based on RAS status that was determined by tissue or plasma testing $[16,17]$.

Using serial ctDNA tracking, two landmark studies independently revealed acquired KRAS mutations as secondary mechanisms of resistance to anti-EGFR therapy with these emerging mutations preceding radiological progression by several months $[18,19]$. One strategy to overcome resistance is the temporary withdrawal of an anti-EGFR antibody, which results in a decline in RASresistant clones and thus opening up an opportunity for an anti-EGFR rechallenge [20]. To highlight the utility of this ctDNA-guided rechallenge strategy, ctDNA analysis was performed in the phase II CRICKET study, which involved rechallenge of patients with RAS and BRAF wild-type $\mathrm{mCRC}$ in the third-line setting who had acquired resistance to first-line cetuximab and irinotecan [21]. In this study, none of the 6 patients who responded to an anti-EGFR rechallenge had RAS mutations found in their plasma, translating into a longer PFS in these patients compared to those with RAS mutated ctDNA at the time of rechallenge (median PFS, 4.0 vs. 1.9 months). The ongoing ctDNA-guided CHRONOS trial (Rechallenge with Panitumumab Driven by RAS Dynamic of Resistance; NCT 03227926) aims to assess the efficacy of initiating panitumumab rechallenge when circulating RAS mutation load decreases by over $50 \%$ compared to the mutation load at the time of progression on first-line anti-EGFR therapy. 


\section{Pancreatic Ductal Adenocarcinoma}

\section{Screening}

Although CA19-9 is used as a serological biomarker for pancreatic ductal adenocarcinoma (PDAC), it is not currently recommended as a screening tool in asymptomatic patients. Mutations in the KRAS are the earliest genetic alteration driving pancreatic neoplasia and are present in $>90 \%$ of PDACs, making it an potential screening biomarker [22]. An early study analysing somatic KRAS mutations in cfDNA in newly diagnosed patients reported detection rates of $42.9 \%$ in stage I and $54.5 \%$ in stage II disease [23]. Other studies have reported sensitivities ranging from 30 to $65 \%[24,25]$. One possible strategy to improve sensitivity is to combine ctDNA detection with other biomarkers, an approach used by Cohen et al. [26] to assess 221 patients with resectable PDAC. Using multiple targets, including elevated serological CA19-9 and a select panel of protein markers along with ctDNA detection of KRAS mutations, they achieved improved sensitivity with this combined biomarker approach compared to any one in isolation. Such an approach could be adopted as a screening tool but larger prospective studies are needed to validate this strategy in a large asymptomatic patient population.

\section{Detection of Minimal Residual Disease}

Our group investigated the prognostic value of preand post-operative KRAS mutant ctDNA detection in 42 patients with resected early-stage PDAC [27]. It is noted that none of these patients had received neoadjuvant treatment and adjuvant treatment was blinded to the ctDNA analysis. Pre-operative detectable ctDNA was associated with an increased risk of recurrence (HR 4.1; 95\% CI 1.8-9.0) and inferior survival (HR 4.1; 95\% CI 1.6-10.5). This suggests that patients with positive pretreatment ctDNA are less likely to be cured by surgery alone and could benefit from more aggressive neoadjuvant therapy. Evidence to support this was demonstrated by Groot et al. [28], who reported that patients with PDAC given neoadjuvant treatment were less likely to have detectable pre-operative ctDNA (21 vs. 69\%). Detection was associated with decreased median RFS ( 8 vs. 19 months; $p<0.001$ ) and poorer survival (14 months vs. not reached, $p<0.001)$. Furthermore, all patients who remained ctDNA positive after neoadjuvant treatment ultimately recurred at a median RFS of 5 months from surgery. This suggests that ctDNA may effectively treat micrometastatic disease; however, what treatment strategies should be adopted if ctDNA was to be present post-neoadjuvant treatment needs to be explored.

Several studies have demonstrated that post-operative ctDNA detection is associated with a shorter time to recurrence $[27,29,30]$. Given this association, the key ques- tion is whether clearance of ctDNA by adjuvant therapy can predict improved outcomes. The currently recruiting DYNAMIC-Pancreas study aims to explore the benefit of intensified adjuvant therapy approach in patients with positive post-operative ctDNA (Table 2 ). The study will randomise patients who have undergone neoadjuvant chemotherapy followed by curative surgery to either a standard of care adjuvant treatment arm or ctDNA-informed arm (ctDNA-negative patients will receive a further 3 months of adjuvant modified FOLIFIRINOX, but those with positive ctDNA will be switched to 4-6 months of adjuvant gemcitabine-based doublet therapy).

\section{Longitudinal Surveillance}

Several studies have demonstrated that ctDNA positivity is an independent prognostic marker of OS in advanced PDACs [31, 32]. Pietrasz et al. [32] measured ctDNA in 104 patients with advanced PDAC, demonstrating that ctDNA detection was associated with poorer survival outcomes (median OS 6.5 vs. 19.0 months, $p<$ 0.001 ) and on multivariate analysis, was an independent prognostic biomarker of OS (HR 3.46; 95\% CI 1.40-8.50). This suggests that ctDNA detection can help identify patients who are likely to have a worse outcome and may benefit from a more intensive approach to their chemotherapy treatment.

ctDNA may also play a role as a biomarker of treatment response. In a cohort of 54 patients receiving chemotherapy for advanced PDAC, increase in KRAS mutant ctDNA predicted disease progression with $83 \%$ sensitivity and $100 \%$ specificity [33]. Rapid changes in ctDNA levels were evident even within 14 days of initiation of chemotherapy and correlated to later radiological response. Importantly, ctDNA had a superior lead time over traditional biomarkers such as CA19-9. Other studies have also demonstrated lead time ranging from 1 to 2 months $[31,34]$. These studies demonstrate that ctDNA allows earlier detection of treatment failure; however, what impacts this should have on treatment decisionmaking remains to be addressed.

\section{Identifying Therapeutic Targets and Resistance}

PDACs are associated with multiple genetic alterations of driver genes such as KRAS, CDKN2A, ERRB2, $W n t, B R C A 1$, and BRCA2 $[35,36]$. Some of these represent potential targets for systemic treatment, for example maintenance olaparib, a PARP inhibitor, recently demonstrated efficacy in metastatic PDACs harbouring germline $B R C A 1$ or $B R C A 2$ mutations [37]. Studies have demonstrated high concordance rates (82-95\%) between ctDNA and tissue mutation detected in patients with PDAC [38]. As proof-of-principle, Patel et al. [39] reported a case of on an 83-year-old man with locally advanced PDAC in whom blood-derived ctDNA revealed 
EGFR G1022S, GNAS R201C, KRAS G12D, MTOR D285fs, and NF1 D1976fs alterations. He was deemed an unsuitable candidate for aggressive chemotherapy and was instead treated with trametinib, potentially targeting the identified GNAS, KRAS, and NF1 alterations. At the time of reporting of this study, the patient remained on treatment at 26+ weeks. Subsequent serial ctDNA could no longer detect EGFR, GNAS, KRAS, and MTOR mutations. This case illustrates the possible role that ctDNA can play in guiding and personalising treatment decision-making.

\section{Upper Gastrointestinal Cancer}

\section{Screening}

Population-based screening for gastric cancers with radiological or endoscopic examination has been implemented in countries with a high prevalence such as Japan and Korea. Given the resource requirements, efforts have been made to develop blood-based screening, but these have yet to be adopted into standard practice.

ctDNA could serve as a serological screening test, but the correlation between ctDNA detection and early-stage gastric cancers is unclear. An analysis of 20 patients with non-metastatic gastric cancer demonstrated that only 4 had detectable ctDNA at baseline [40]. The CancerSeek platform, combining ctDNA detection with protein biomarkers, reported improved sensitivity rates of 73 and $70 \%$ in non-metastatic gastric and oesophageal cancers, suggesting one strategy to improve detection [8]. Lin et al. [41] also adopted a tumour-agnostic approach, utilising methylation rates of three previously identified gastric cancer-related genes, ZIC1, HOXD10, and RUNX3. In a series of patients with early and advanced gastric cancer as well as intestinal metaplasia, intraepithelial neoplasia, and healthy controls, methylation rates increased significantly from normal controls to pre-invasive lesions and gastric cancer. Of note, higher methylation rates of $H O X D 10$ were found in advanced versus early gastric cancers. This early trial provides tantalising data in regards to how ctDNA could be used to not only detect early gastric cancers but also monitor pre-cancerous lesions.

\section{Detection of Minimal Residual Disease}

The utility of ctDNA in upper gastrointestinal (UGI) cancers as a prognostic biomarker post-surgery has been explored. Maron et al. [42] measured the variant allele fraction (VAF) of somatic alterations in plasma from 1,630 patients diagnosed with oesophageal, gastroesophageal junction (GOJ), or gastric adenocarcinoma. Patients with positive post-surgery ctDNA, using a VAF detection cut-off of $0.25 \%$, had worse DFS than those with negative ctDNA (median 2.5 months vs. unreached, $p=0.03$ ). A smaller study reported that detectable postsurgery ctDNA predicted for relapse in 6 of 8 patients with a median lead time of 4.05 months to clinical recurrence [43]. A further study investigating ctDNA dynamics in 11 patients with gastric cancer reported that cfDNA levels were initially elevated in the first 3 weeks post-surgery but subsequently declined over the next 3 to 4 months [44]. This emphasises that the optimal timing of ctDNA testing needs to be further defined. Future studies that incorporate post-surgical ctDNA status into treatment selection in the adjuvant setting, ideally with a randomised design compared to standard of care, are needed to confirm the utility of ctDNA in improving treatment decision-making in patients with resected UGI cancers.

Given that neoadjuvant treatment is standard for almost all potentially resectable UGI cancers, an important question is whether ctDNA detected after neoadjuvant treatment predicts relapse post-surgery. Azad et al. [45] analysed post-chemoradiotherapy plasma in early-stage oesophageal cancer patients and found that 5 out of 31 were positive for ctDNA. These patients were found to be at a significantly increased risk of disease progression (HR 18.7; 95\% CI 1.1-316.5), distant metastases (HR 32.1; 95\% CI 1.8-559.2), and disease-specific death (HR 23.1; 95\% CI 2.0-273.5) compared to ctDNA-negative patients. However, it must be noted that all 5 of these patients were treated with definitive chemoradiotherapy alone and did not undergo surgery. Based on this early evidence, it appears that ctDNA positivity post-initial chemoradiation is associated with a high relapse risk. How this risk should be addressed needs to explored in further studies.

\section{Longitudinal Surveillance}

Routine monitoring of CEA and CA19-9 during treatment is not recommended in published guidelines $[46,47]$. Early data suggests that routine ctDNA monitoring could be developed to meet this important unmet need. Normando et al. [48] performed 3-monthly serial ctDNA testing, based on the ratio of expression of Alu DNA sequences (short DNA stretches associated with genomic instability) to total cfDNA, in patients with unresectable or advanced gastric cancer receiving systemic chemotherapy. In the 20 patients with serial ctDNA available for analysis, elevated levels of ctDNA detected 3 months after the start of chemotherapy was significantly correlated with poorer DFS $(p=0.022)$. Another study in patients with metastatic oesophageal/GOJ and gastric adenocarcinoma stratified patients as having high or low plasma VAF [43]. They hypothesised that a high maximal tumour somatic VAF (maxVAF) would reflect higher tumour burden and therefore be associ- 
ated with worst outcomes. Indeed, there appeared to be a trend to worse outcomes in high versus low patients (median OS 9.4 vs. 14.8 months, $p=0.1$ ). They then analysed ctDNA dynamics to predict response to treatment in 25 patients that had available longitudinal ctDNA within their first 150 days of treatment [43]. A decline of $\geq 50 \%$ in maxVAF was associated with longer survival (median survival 13.7 vs. 8.6 months, $p=0.02$ ), demonstrating that ctDNA monitoring could predict treatment response.

\section{Identifying Therapeutic Targets and Resistance}

Standard therapeutic targets for advanced gastric, oesophageal, and GOJ cancers include HER2 and PDL1. Wang et al. [49] performed HER2 evaluation on matched tumour tissue (via dual in situ hybridisation) and plasma from 56 patients with advanced gastric cancer demonstrating high concordance (97.1\%). They also reported plasma HER 2 copy numbers appeared to correlate with treatment response, decreasing when patients were responding to treatment and then increasing when disease progression occurred. A later study reported that increasing tumour burden index, a measure of the percentage of ctDNA targets in plasma, predicted for progression in patients with advanced gastric cancer receiving trastuzumab [50]. Further analysis of paired baseline and time-of-progression ctDNA in 10 patients also revealed emergence of 86 secondary mutations. The key molecular pathways most frequently altered by these mutations were MAPK and EGFR, revealing that mechanisms of drug resistance are multifaceted and heterogeneous. These studies demonstrate that ctDNA interrogation of HER2 status can not only identify patients that would benefit from targeted therapy but also allow early evaluation of response and resistance without the need for repeat sampling of tumour tissue.

The use of PD-L1 overexpression as a biomarker to predict response to immunotherapy has been demonstrated to be unreliable across many cancer types, as even tumours with low levels of expression can have pronounced and prolonged responses. Therefore, more robust markers are required. Kim et al. [51] performed molecular characterisation of tissue and ctDNA from $61 \mathrm{pa}-$ tients with metastatic gastric cancer receiving pembrolizumab. Patients with higher ctDNA mutation load at baseline appeared to have significantly improved overall response rates $(83 \% ; p<0.01)$. Change in ctDNA 6 weeks post-treatment also appeared to predict response, where all 4 patients who had increasing ctDNA experienced progression within 100 days, whereas those with decreasing ctDNA showed improvements in ORR (58 vs. $0 \% ; p=0.048$ ) and PFS (median 123 vs. 66 days; $p=$ 0.029 ). This evidence needs to be validated in larger trials but if confirmed, could lead to the development of a reliable method of predicting and measuring response to immunotherapy not only for UGI cancers but other cancer types as well.

\section{Conclusion}

ctDNA analysis represents an exciting and dynamic biomarker for gastrointestinal cancers. It has been demonstrated to be a marker of minimal residual disease after initial definitive treatment and offers prognostic information in both localised and advanced disease. However, further work is needed to determine how detection should shape treatment decision-making. Several ongoing randomised clinical trials are currently exploring the clinical utility of ctDNA-guided strategies in the adjuvant setting, which include escalating treatment in ctDNA-positive patients and de-escalating treatment in ctDNA-negative patients (Table 2). Consideration should also be given to even earlier treatment adaptation, perhaps avoiding surgery and continuing with systemic treatment in patients with positive ctDNA post-neoadjuvant treatment given the high risk of systemic relapse. Outcomes in patients could also be improved if ctDNA could be adopted as a screening tool and also employed to identify therapeutic targets and interrogate methods of treatment resistance. It is clear that further prospective trials are needed to explore all of these applications, but ctDNA clearly has substantial potential as a clinically useful marker in the management of gastrointestinal cancers from cancer screening through to treatment of advanced disease.

\section{Conflict of Interest Statement}

The authors have no conflicts of interest to declare.

\section{Funding Sources}

No funding was necessary for the writing of this paper since the work is a literature review.

\section{Author Contributions}

Y.H.T., J.T., and B.L.: conceptualisation. Y.H.T.: writing - original draft preparation. All authors: writing - review and editing. Y.H.T.: final revisions. All authors have approved the final version of the manuscript. 


\section{References}

1 Stroun M, Anker P, Maurice P, Lyautey J, Lederrey C, Beljanski M. Neoplastic characteristics of the DNA found in the plasma of cancer patients. Oncology. 1989;46(5):31822.

2 Wan JC, Massie C, Garcia-Corbacho J, Mouliere F, Brenton JD, Caldas C, et al. Liquid biopsies come of age: towards implementation of circulating tumour DNA. Nat Rev Cancer. 2017 Apr;17(4):223-38.

3 Elazezy M, Joosse SA. Techniques of using circulating tumor DNA as a liquid biopsy component in cancer management. Comput Struct Biotechnol J. 2018 Oct;16:370-8.

4 Health and welfare overview. Analysis of cancer outcomes and screening behaviour for national cancer screening programs in Australia. 2018, AIHW: Canberra.

5 Adler, A., Geiger S, Keil A, Bias H, Schatz P, deVos $\mathrm{T}$, et al. Improving compliance to colorectal cancer screening using blood and stool based tests in patients refusing screening colonoscopy in Germany. BMC Gastroenterol. 2014;14:183.

6 Tie J, Wang Y, Kinde I, Steel M, Elsaeh H, Sing MS, et al. Circulating tumor DNA (ctDNA) in nonmetastatic colorectal cancer (CRC): Potential role as a screening tool. J Clin Oncol. 2015.33(3_suppl):518.

7 Bettegowda C, Sausen M, Leary RJ, Kinde I, Wang Y, Agrawal N, et al. Detection of circulating tumor DNA in early- and late-stage human malignancies. Sci Transl Med. 2014 Feb; 6(224):224ra24.

8 Cohen JD, Li L, Wang Y, Thorburn C, Afsari B, Danilova L, et al. Detection and localization of surgically resectable cancers with a multianalyte blood test. Science. 2018 Feb; 359(6378):926-30.

9 Tie J, Wang Y, Tomasetti C, Li L, Springer S, Kinde I, et al. Circulating tumor DNA analysis detects minimal residual disease and predicts recurrence in patients with stage II colon cancer. Sci Transl Med. 2016 Jul;8(346): 346 ra92.

10 Tie J, Cohen JD, Wang Y, Li L, Christie M, Simons K, et al. Serial circulating tumour DNA analysis during multimodality treatment of locally advanced rectal cancer: a prospective biomarker study. Gut. 2019 Apr; 68(4):663-71.

11 Reinert T, Henriksen TV, Christensen E, Sharma S, Salari R, Sethi H, et al. Analysis of Plasma Cell-Free DNA by Ultradeep Sequencing in Patients With Stages I to III Colorectal Cancer. JAMA Oncol. 2019 May; 5(8): 1124.

12 Tie J, Cohen JD, Wang Y, Christie M, Simons $\mathrm{K}$, Lee $\mathrm{M}$, et al. Circulating Tumor DNA Analyses as Markers of Recurrence Risk and Benefit of Adjuvant Therapy for Stage III Colon Cancer. JAMA Oncol. 2019 Oct;5(12): 1710-7.

13 Tie J, Kinde I, Wang Y, Wong HL, Roebert J, Christie M, et al. Circulating tumor DNA as an early marker of therapeutic response in patients with metastatic colorectal cancer. Ann Oncol. 2015 Aug;26(8):1715-22.
14 Lyskjær I, Kronborg CS, Rasmussen MH, Sørensen BS, Demuth C, Rosenkilde $\mathrm{M}$, et al. Correlation between early dynamics in circulating tumour DNA and outcome from FOLFIRI treatment in metastatic colorectal cancer. Sci Rep. 2019 Aug;9(1):11542.

15 Bachet JB, Bouché O, Taieb J, Dubreuil O, Garcia ML, Meurisse A, et al. RAS mutation analysis in circulating tumor DNA from patients with metastatic colorectal cancer: the AGEO RASANC prospective multicenter study. Ann Oncol. 2018 May;29(5):1211-9.

16 Grasselli J, Elez E, Caratù G, Matito J, Santos C, Macarulla T, et al. Concordance of bloodand tumor-based detection of RAS mutations to guide anti-EGFR therapy in metastatic colorectal cancer. Ann Oncol. 2017 Jun;28(6): 1294-301.

17 Vidal J, Muinelo L, Dalmases A, Jones F, Edelstein D, Iglesias $\mathrm{M}$, et al. Plasma ctDNA RAS mutation analysis for the diagnosis and treatment monitoring of metastatic colorectal cancer patients. Ann Oncol. 2017 Jun;28(6): 1325-32.

18 Misale S, Di Nicolantonio F, Sartore-Bianchi A, Siena S, Bardelli A. Resistance to anti-EGFR therapy in colorectal cancer: from heterogeneity to convergent evolution. Cancer Discov. 2014 Nov;4(11):1269-80.

19 Diaz LA Jr, Williams RT, Wu J, Kinde I, Hecht JR, Berlin J, et al. The molecular evolution of acquired resistance to targeted EGFR blockade in colorectal cancers. Nature. 2012 Jun; 486(7404):537-40.

20 Parseghian CM, Loree JM, Morris VK, Liu X, Clifton KK, Napolitano S, et al. Anti-EGFRresistant clones decay exponentially after progression: implications for anti-EGFR re-challenge. Ann Oncol. 2019 Feb;30(2):243-9.

21 Cremolini C, Antoniotti C, Lonardi S, Aprile G, Bergamo F, Masi G, et al. Activity and Safety of Cetuximab Plus Modified FOLFOXIRI Followed by Maintenance With Cetuximab or Bevacizumab for RAS and BRAF Wildtype Metastatic Colorectal Cancer: A Randomized Phase 2 Clinical Trial. JAMA Oncol. 2018 Apr;4(4):529-36.

22 Kanda M, Matthaei H, Wu J, Hong SM, Yu J, Borges M, et al. Presence of somatic mutations in most early-stage pancreatic intraepithelial neoplasia. Gastroenterology. 2012 Apr; 142(4):730-733.e9.

23 Lee B, Cohen J, Lipton LR, Tie J, Javed AA, Lu $\mathrm{L}$, et al. Potential role of circulating tumor DNA (ctDNA) in the early diagnosis and post-operative management of localised pancreatic cancer. J Clin Oncol. 2017. 35(15_suppl):4101.

24 Sefrioui D, Blanchard F, Toure E, Basile P, Beaussire L, Dolfus C, et al. Diagnostic value of CA19.9, circulating tumour DNA and circulating tumour cells in patients with solid pancreatic tumours. Br J Cancer. 2017 Sep; 117(7):1017-25.

25 Wang ZY, Ding XQ, Zhu H, Wang RX, Pan XR, Tong JH. KRAS Mutant Allele Fraction in Circulating Cell-Free DNA Correlates With Clinical Stage in Pancreatic Cancer Patients. Front Oncol. 2019 Nov; 9:1295.
26 Cohen JD, Javed AA, Thoburn C, Wong F, Tie J, Gibbs P, et al. Combined circulating tumor DNA and protein biomarker-based liquid biopsy for the earlier detection of pancreatic cancers. Proc Natl Acad Sci USA. 2017 Sep; 114(38):10202-7.

27 Lee B, Lipton L, Cohen J, Tie J, Javed AA, Li $\mathrm{L}$, et al. Circulating tumor DNA as a potential marker of adjuvant chemotherapy benefit following surgery for localized pancreatic cancer. Ann Oncol. 2019 Sep;30(9):1472-8.

28 Groot VP, Mosier S, Javed AA, Teinor JA, Gemenetzis G, Ding D, et al. Circulating Tumor DNA as a Clinical Test in Resected Pancreatic Cancer. Clin Cancer Res. 2019 Aug; 25(16):4973-84.

29 Bernard V, Kim DU, San Lucas FA, Castillo J, Allenson K, Mulu FC, et al. Circulating Nucleic Acids Are Associated With Outcomes of Patients With Pancreatic Cancer. Gastroenterology. 2019 Jan;156(1):108-18.e4.

30 Hadano N, Murakami Y, Uemura K, Hashimoto Y, Kondo N, Nakagawa N, et al. Prognostic value of circulating tumour DNA in patients undergoing curative resection for pancreatic cancer. Br J Cancer. 2016 Jun; 115(1):59-65.

31 Park G, Park JK, Son DS, Shin SH, Kim YJ, Jeon HJ, et al. Utility of targeted deep sequencing for detecting circulating tumor DNA in pancreatic cancer patients. Sci Rep. 2018 Aug;8(1):11631.

32 Pietrasz D, Pécuchet N, Garlan F, Didelot A, Dubreuil O, Doat S, et al. Plasma Circulating Tumor DNA in Pancreatic Cancer Patients Is a Prognostic Marker. Clin Cancer Res. 2017 Jan;23(1):116-23.

33 Kruger S, Heinemann V, Ross C, Diehl F, Nagel D, Ormanns $S$, et al. Repeated mutKRAS ctDNA measurements represent a novel and promising tool for early response prediction and therapy monitoring in advanced pancreatic cancer. Ann Oncol. 2018 Dec;29(12): 2348-55.

34 Tjensvoll K, Lapin M, Buhl T, Oltedal S, Steen-Ottosen Berry K, Gilje B, et al. Clinical relevance of circulating KRAS mutated DNA in plasma from patients with advanced pancreatic cancer. Mol Oncol. 2016 Apr;10(4): 635-43.

35 Murphy SJ, Hart SN, Lima JF, Kipp BR, Klebig $\mathrm{M}$, Winters JL, et al. Genetic alterations associated with progression from pancreatic intraepithelial neoplasia to invasive pancreatic tumor. Gastroenterology. 2013 Nov; 145(5): 1098-109.e1.

36 Iqbal J, Ragone A, Lubinski J, Lynch HT, Moller P, Ghadirian P, et al.; Hereditary Breast Cancer Study Group. The incidence of pancreatic cancer in BRCA 1 and BRCA $2 \mathrm{mu}$ tation carriers. Br J Cancer. 2012 Dec;107(12): 2005-9.

37 Golan T, Varadhachary GR, Sela T, Fogelman DR, Halperin N, Shroff T, et al. Phase II study of olaparib for BRCAness phenotype in pancreatic cancer. J Clin Oncol. 2018;36(4_suppl):297. 
38 Berger AW, Schwerdel D, Ettrich TJ, Hann A, Schmidt SA, Kleger A, et al. Targeted deep sequencing of circulating tumor DNA in metastatic pancreatic cancer. Oncotarget. 2017 Dec;9(2):2076-85.

39 Patel H, Okamura R, Fanta P, Patel C, Lanman RB, Raymond VM, et al. Clinical correlates of blood-derived circulating tumor DNA in pancreatic cancer. J Hematol Oncol. 2019 Dec;12(1): 130 .

40 Cabel L, Decraene C, Bieche I, Pierga JY, Bennamoun M, Fuks D, et al.; Limited Sensitivity of Circulating Tumor DNA Detection by Droplet Digital PCR in Non-Metastatic Operable Gastric Cancer Patients. Limited Sensitivity of Circulating Tumor DNA Detection by Droplet Digital PCR in Non-Metastatic Operable Gastric Cancer Patients. Cancers (Basel). 2019 Mar;11(3):E396.

41 Lin Z, Luo M, Chen X, He X, Qian Y, Lai S, et al. Combined Detection of Plasma ZIC1, HOXD10 and RUNX3 Methylation is a Promising Strategy for Early Detection of Gastric Cancer and Precancerous Lesions. J Cancer. 2017 Apr;8(6):1038-44.

42 Maron SB, Chase LM, Lomnicki S, Kochanny S, Moore KL, Joshi SS, et al. Circulating Tumor DNA Sequencing Analysis of Gastroesophageal Adenocarcinoma. Clin Cancer Res. 2019 Dec;25(23):7098-112.

43 Kim YW, Kim YH, Song Y, Kim HS, Sim HW, Poojan S, et al. Monitoring circulating tumor DNA by analyzing personalized cancer-specific rearrangements to detect recurrence in gastric cancer. Exp Mol Med. 2019 Aug;51(8): $1-10$.

44 Pu WY, Zhang R, Xiao L, Wu YY, Gong W, Lv XD, et al. Prediction of cancer progression in a group of 73 gastric cancer patients by circulating cell-free DNA. BMC Cancer. 2016 Dec;16(1):943.
45 Azad TD, Chaudhuri AA, Fang P, Qiao Y, Esfahani MS, Chabon JJ, et al. Circulating Tumor DNA Analysis for Detection of Minimal Residual Disease After Chemoradiotherapy for Localized Esophageal Cancer. Gastroenterology. $2020 \mathrm{Feb} ; 158(3)$ :494-505.e6.

46 Ajani JA, D’Amico TA, Almhanna K, Bentrem DJ, Chao J, Das P, et al. Gastric Cancer, Version 3.2016, NCCN Clinical Practice Guidelines in Oncology. J Natl Compr Canc Netw. 2016 Oct;14(10):1286-312.

47 Ajani JA, D’Amico TA, Bentrem DJ, Chao J, Corvera C, Das P, et al. Esophageal and Esophagogastric Junction Cancers, Version 2.2019, NCCN Clinical Practice Guidelines in Oncology. J Natl Compr Canc Netw. 2019 Jul; 17(7):855-83

48 Normando SR, Delgado PO, Rodrigues AK, David Filho WJ, Fonseca FL, Cruz FJ, et al. Circulating free plasma tumor DNA in patients with advanced gastric cancer receiving systemic chemotherapy. BMC Clin Pathol. 2018 Nov; 18(1): 12.

49 Wang H, Li B, Liu Z, Gong J, Shao L, Ren J, et al. HER2 copy number of circulating tumour DNA functions as a biomarker to predict and monitor trastuzumab efficacy in advanced gastric cancer. Eur J Cancer. 2018 Jan;88:92-100.

50 Wang Y, Zhao C, Chang L, Jia R, Liu R, Zhang $\mathrm{Y}$, et al. Circulating tumor DNA analyses predict progressive disease and indicate trastuzumab-resistant mechanism in advanced gastric cancer. EBioMedicine. 2019 May;43:261-9.

51 Kim ST, Cristescu R, Bass AJ, Kim KM, Odegaard JI, Kim K, et al. Comprehensive molecular characterization of clinical responses to PD-1 inhibition in metastatic gastric cancer. Nat Med. 2018 Sep;24(9):1449-58. 